\title{
Elderly human hematopoietic progenitor cells express cellular senescence markers and are more susceptible to pyroptosis
}

\author{
Tinhinane Fali, ${ }^{1}$ Véronique Fabre-Mersseman, ${ }^{1}$ Takuya Yamamoto, ${ }^{2,3,4}$ Charles Bayard, ${ }^{1}$ \\ Laura Papagno, ${ }^{1}$ Solène Fastenackels, ${ }^{1}$ Rima Zoorab, ${ }^{1}$ Richard A. Koup, ${ }^{2}$ Jacques Boddaert, ${ }^{5}$ \\ Delphine Sauce, ${ }^{1}$ and Victor Appay ${ }^{1,6}$ \\ 'Sorbonne Université, INSERM, Centre d'Immunologie et des Maladies Infectieuses (CIMI-Paris), Paris, France. \\ ${ }^{2}$ Immunology Laboratory, Vaccine Research Center, National Institute of Allergy and Infectious Diseases, NIH, Bethesda, \\ Maryland, USA. ${ }^{3}$ Laboratory of Immunosenescence, National Institutes of Biomedical Innovation, Health and Nutrition, \\ Ibaraki-City, Osaka, Japan. ${ }^{4}$ Center for AIDS Research, Kumamoto University, Kumamoto, Japan. \\ ${ }^{5}$ AP-HP, Hôpital Pitié-Salpêtrière, Service de Gériatrie, Paris, France. ${ }^{6}$ International Research Center of Medical Sciences \\ (IRCMS), Kumamoto University, Kumamoto, Japan.
}

The maintenance of effective immunity over time is dependent on the capacity of hematopoietic stem cells (HSCs) to sustain the pool of immunocompetent mature cells. Decline of immune competence with old age may stem from HSC defects, including reduced self-renewal potential and impaired lymphopoiesis, as suggested in murine models. To obtain further insights into aging-related alteration of hematopoiesis, we performed a comprehensive study of blood hematopoietic progenitor cells (HPCs) from older humans. In the elderly, HPCs present active oxidative phosphorylation and are pressed to enter cell cycling. However, p53-p21 and p15 cell senescence pathways, associated with telomerase activity deficiency, strong telomere attrition, and oxidative stress, are engaged, thus limiting cell cycling. Moreover, survival of old HPCs is impacted by pyroptosis, an inflammatory form of programmed cell death. Lastly, telomerase activity deficiency and telomere length attrition of old HPCs may be passed on to progeny cells such as naive T lymphocytes, further highlighting the poor hematopoietic potential of the elderly. This presenescent profile is characteristic of the multiple intrinsic and extrinsic factors affecting HPCs in elderly individuals and represents a major obstacle in terms of immune reconstitution and efficacy with advanced age.

Authorship note: DS and VA contributed equally to this work.

Conflict of interest: The authors have declared that no conflict of interest exists.

Submitted: May 23, 2017

Accepted: May 31, 2018

Published: July 12, 2018

Reference information: JCI Insight. 2018;3(13):e95319. https://doi.org/10.1172/jci. insight.95319.

\section{Introduction}

Aging is associated with an increased prevalence of multiple comorbidities, including infectious and malignant diseases. Many of these disorders are thought to stem from old-age-related immune decline. The immune system is composed of a multitude of distinct immunocompetent cells, with specialized functions, that are regrouped into the adaptive (i.e., T and B lymphocytes) or innate (i.e., myeloid cell types, mast cells, natural killer cells, and dendritic cells) arms of immunity. Increasing efforts to characterize the immune system of elderly people in recent years have revealed that most immunocompetent cell compartments present profound quantitative as well as qualitative impairments. The cause of these impairments can vary, and is often related to the exhaustion of the cells or their functions over time in inflammatory settings (1). The majority of mature blood cell compartments need, therefore, to be continuously replenished or replaced, which is the role of hematopoietic progenitor cells (HPCs) and, ultimately, hematopoietic stem cells (HSCs). While the self-renewal and differentiation potential of stem cells, along with their blood cell reconstitution capacity, have long been considered as infinite, increasing evidence indicates that this is not the case. Under conditions of stress, HSCs eventually exhibit several functional defects, including a diminished regenerative and self-renewal potential (2). Loss of stem cell activity is therefore a likely mechanism of impairment common to many mature cell types, thus representing a central cause of immune-competence decline.

Most studies on HSC aging have been carried out in mouse models, and have highlighted extrinsic and intrinsic factors affecting the function of HSCs. Gene expression profiling of purified HSCs from young 
and old mice revealed a general downregulation of genes involved in lymphopoiesis and an upregulation of myeloid genes in aged HSCs, consistent with an aging-associated differentiation skewing $(3,4)$. It has also been shown that HSCs from aged mice present more $\gamma \mathrm{H} 2 \mathrm{AX}$ foci, used as a surrogate marker for DNA damage (5-8), which is a debated cause of HSC functional impairment (9). A recent study reveals that loss of autophagy in most HSCs from aged mice causes an activated metabolic state, which is associated with accelerated myeloid differentiation, and impairs HSC self-renewal activity and regenerative potential (10). In humans, much less information is available on the aged HSCs, due to the limited and challenging access to bone marrow samples of elderly humans, the niche of HSCs. Reduced transplantation success in patients receiving HSCs isolated from older (45 years and above) donor bone marrows indicates that human HSC regenerative capacity also declines with aging (11). Isolated studies of aged human bone marrow samples also confirmed the altered lymphoid differentiation potential of HSCs (12-14), and accumulation of DNA damage in these cells (15). Gain of new knowledge to further our understanding of HSC or HPC impairments and their impact on hematopoietic capacity with aging is a priority, particularly in humans.

We performed here a comprehensive study of blood HPCs, as an alternative to bone marrow HSCs, to overcome the constraint of sample availability from elderly adults. Based on phenotypic analyses, in vitro T lymphocyte differentiation assays, and gene expression profiling of circulating HPCs from aged subjects, we demonstrate impaired lymphopoiesis and active cell cycling of HPCs with aging, and provide insights into their functional impairments. Our findings reveal that, while mobilized, elderly HPCs present evidence of cellular senescence and increased cell death by pyroptosis. Reduced telomere length and telomerase activity in old HPCs may affect the properties of their progeny, such as mature T lymphocytes.

\section{Results}

Reduced lymphopoietic capacity of blood HPCs from the elderly. HPCs were identified as being CD34 ${ }^{+} \mathrm{Lin}^{-}$ CD45 $5^{\mathrm{dim}}$ cells in human blood (Figure 1A), and were in the range of 1 to 10 cells per $\mu$ of blood of young healthy subjects, which makes them relatively rare events compared with bone marrow HSCs or HPCs. The enumeration of these cells revealed that elderly individuals present a significant reduction in circulating $\mathrm{CD}_{3} 4^{+}$cell numbers (Figure 1B). We then assessed the phenotypic distribution of circulating HPCs. In mice, HSC subpopulations with distinct lineage potential (e.g., lymphoid or myeloid biased) have been identified and coexist in the bone marrow to coordinate hematopoiesis (16-19). The phenotypic dissection of HPCs into well-defined subsets is still at an early stage in humans. However, several studies concur with the distinction between $\mathrm{CD} 38^{+} \mathrm{CD} 117^{-} \mathrm{CD} 45 \mathrm{RA}^{+} \mathrm{CD} 10^{+} \mathrm{HPCs}$ with lymphoid precursor properties, versus $\mathrm{CD}^{2} 8^{+} \mathrm{CD} 117^{+} \mathrm{CD} 45 \mathrm{RA}^{-} \mathrm{CD} 10^{-}$HPCs with myeloid precursor properties, usually defined as common lymphoid progenitors (CLPs) or common myeloid progenitors (CMPs), respectively (20-23). In the blood, the majority of circulating $\mathrm{CD} 34^{+} \mathrm{Lin}^{-}$cells appeared to be $\mathrm{CD} 90^{-} \mathrm{CD} 38^{+}$committed HPCs, which could be separated into CLPs and CMPs based on these markers (Figure 1C). Decreasing CLP to CMP ratios were observed in the elderly compared with younger subjects (Figure 1D). Circulating CLP numbers were particularly reduced with old age (Figure 1E). This suggests that the age-associated disruption of HPCs affects particularly lymphopoiesis, in line with observations from mouse $(3,24)$ or human $(12)$ bone marrow samples.

In order to further address this issue at the functional level, we tested the potential of circulating old $\mathrm{CD}_{3}{ }^{+}$cells to enter the T lymphocyte lineage differentiation pathway, using the OP9-DL1 coculture experimental system. Equivalent numbers of purified circulating $\mathrm{CD} 34^{+}$cells from aged or young subjects were thus cultured with the OP9-DL1 stromal cell line, expressing the T cell differentiation-related notch ligand. The in vitro generation of $\mathrm{CD} 34^{+} \mathrm{CD} 45 \mathrm{RA}^{+} \mathrm{CD} 7^{+} \mathrm{T}$ lymphocyte precursors (TLPs) as well as their distribution into pro- and pre-T subsets were assessed after 7, 14, 21, and 28 days of coculture by flow cytometry based on the expression of standard phenotypic markers (Supplemental Figure 1; supplemental material available online with this article; https://doi.org/10.1172/jci.insight.95319DS1). Compared with HPCs from young subjects, old HPCs yielded lower proportions and absolute counts of TLPs in culture (Figure 1, F and G). Distribution of TLPs from young HPCs showed a steady evolution in culture, from a more pro$\mathrm{T} 1\left(\mathrm{CD} 5^{-} \mathrm{CD} 1 \mathrm{a}^{-}\right)$to a more pre-T1 $\left(\mathrm{CD}^{+} \mathrm{CD} 1 \mathrm{a}^{+}\right)$phenotype, as expected from the $\mathrm{T}$ lymphocyte differentiation of progenitors in this system (Figure 1H). In contrast, TLPs generated from old HPCs presented an early and steady bias towards more differentiated pre-T1 cells (Figure 1, H and I), suggesting an active pretuned state of differentiation. On the whole, phenotypic and functional analyses of circulating HPCs from aged individuals point towards qualitative defects of these cells, affecting in particular lymphopoiesis and the generation of $\mathrm{T}$ lymphocytes. 
A

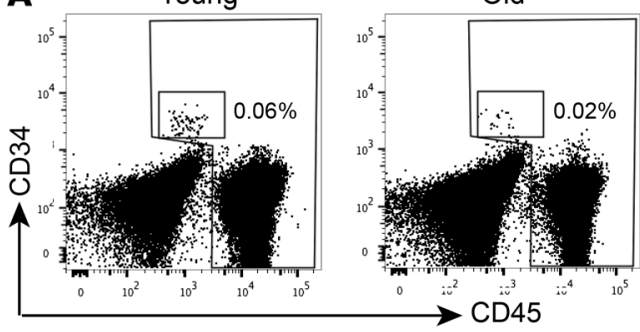

C

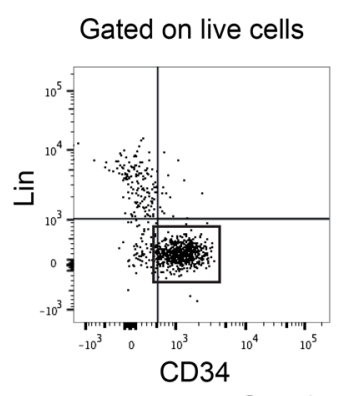

Gated on CD34+ Lin-
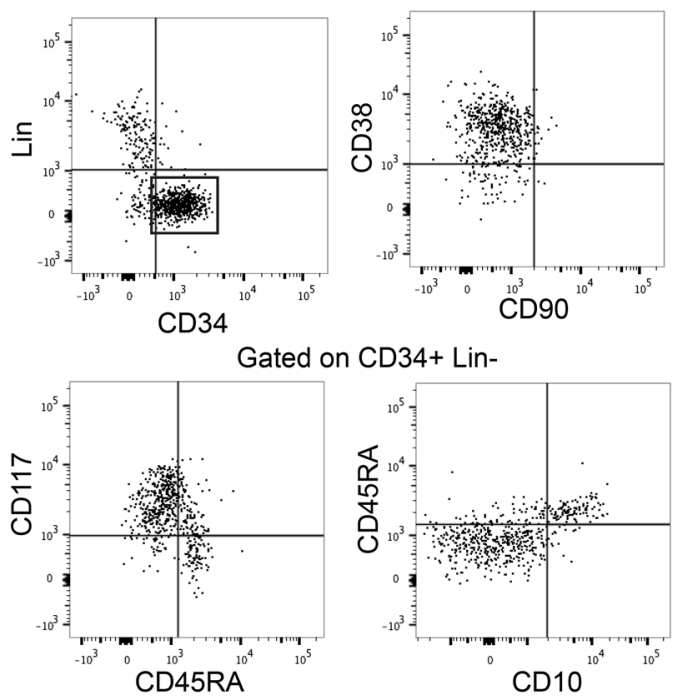

B

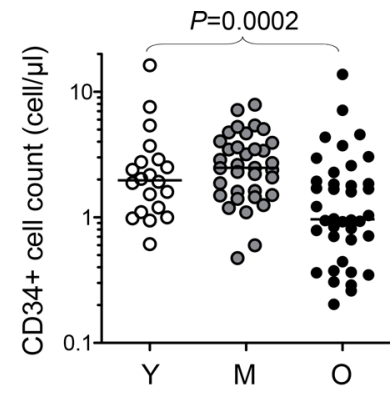

D

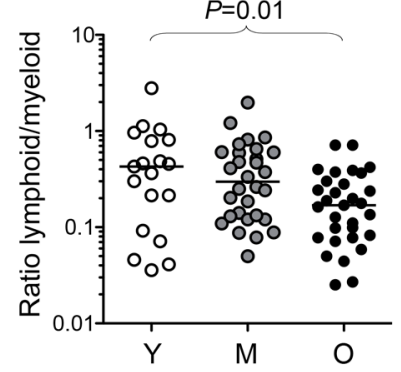

F 257

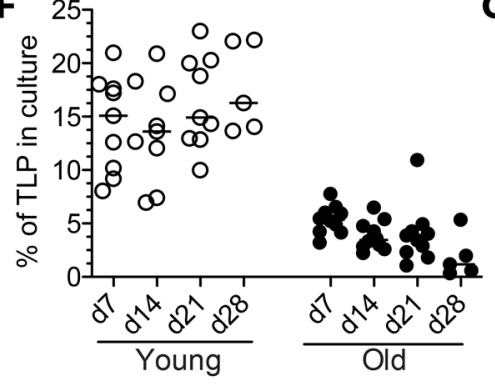

E
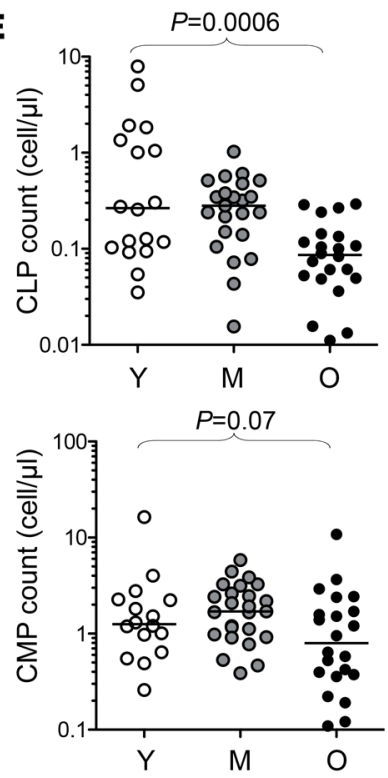

G

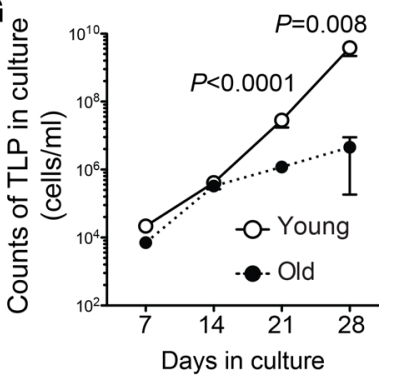

H

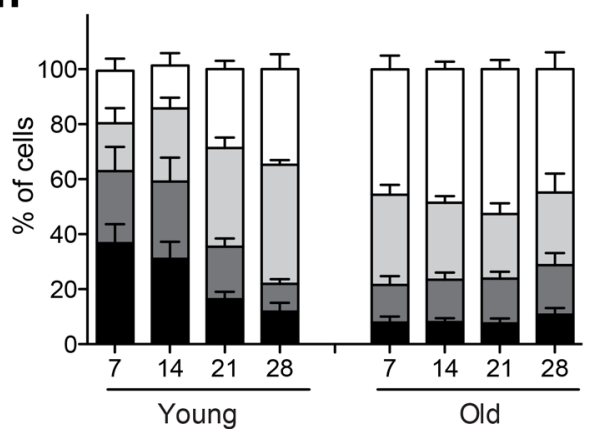

I

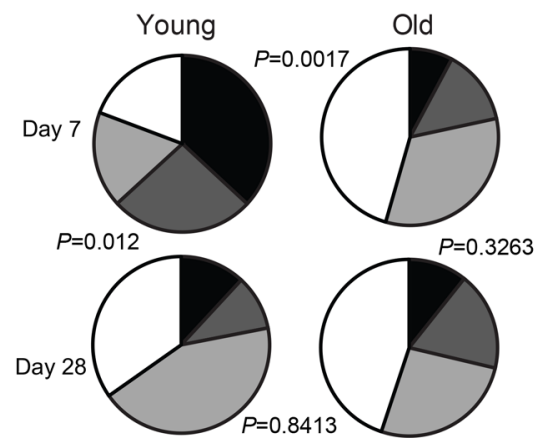

Figure 1. Altered lymphopoietic capacity of circulating CD34+ hematopoietic progenitors from elderly humans. (A) Representative examples of CD34 and CD45 staining to identify HPCs within the CD45 ${ }^{10}$ population. (B) Absolute counts of CD34 ${ }^{+} C D 45^{10} L^{2} n^{-}$cells in young (Y, $\left.n=20\right)$, middleaged $(M, n=35)$, or old $(0, n=40)$ healthy adults. (C) Representative staining for CD38, CD90, CD117, CD45RA, and CD10 on bead-enriched CD34+ cells from PBMCs of a healthy adult. (D) Ratio of common lymphoid progenitors (CLPs, CD38+CD117-CD45RA ${ }^{+}$CD10+) versus common myeloid progenitors (CMPs, CD38 ${ }^{+}$CD117 + CD45RA-CD10-) within $C D 34^{+}$cells from PBMCs in young, middle-aged, or old healthy adults. (E) Frequency of CLPs or CMPs in the blood of young, middle-aged, or old healthy adults. (F) Frequency of TLPs upon in vitro differentiation of FACS-isolated CD34 ${ }^{+}$HPCs from young $(n=9)$ or old $(n=10)$ healthy adults. Phenotyping of CD34+ cells was performed after 7, 14, 21, and 28 days in the OPg-DL1 coculture system. (C) Mean absolute counts of TLPs in culture upon in vitro differentiation of CD34+ HPCs purified from young $(n=9)$ or old $(n=10)$ healthy adults in the OP9-DL1 coculture system. (H) Distribution of TLP subsets of differentiation (ProT1: CD45RA+CD7+CD5-CD1a-; ProT2: CD45RA CD7+CD5 ${ }^{+}$CD1a-;

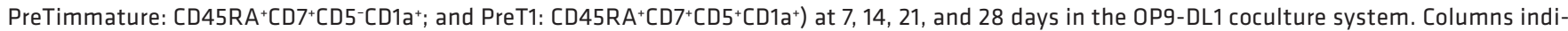
cate mean values (+SEM). (I) Percentages of TLP subsets within the total population in vitro are represented in pie charts for simplicity (black slices correspond to proT1, dark gray to proT2, light gray to preTimmature, and white to preT1). Pies show mean values. The Mann-Whitney or KruskallWallis test was used for comparing 2 or 3 groups, respectively. Bars indicate the median. 
A
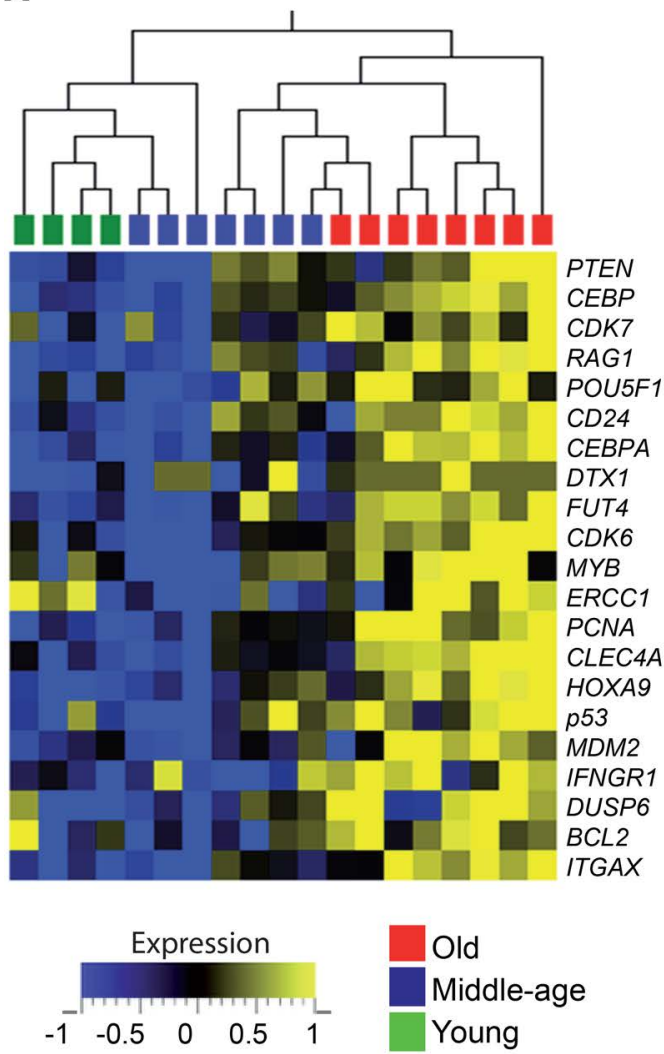

B

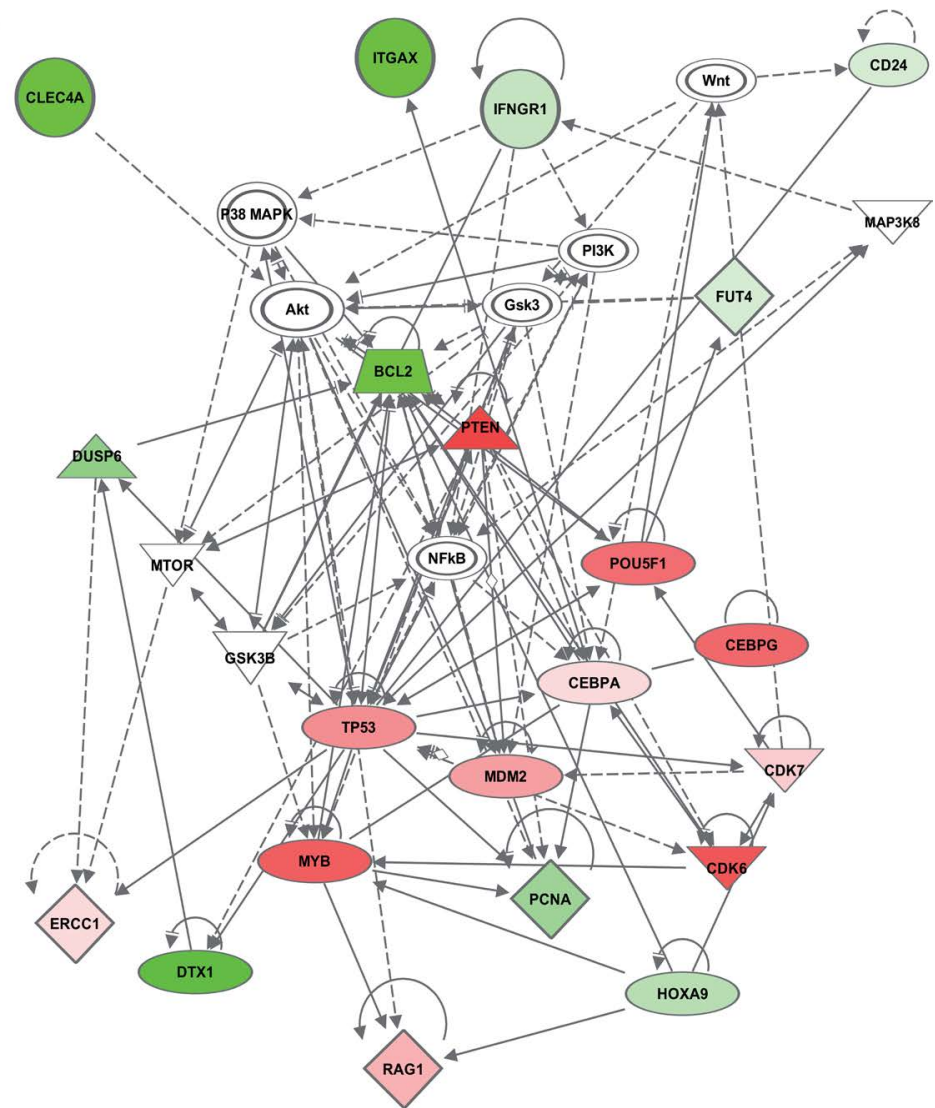

Figure 2. Transcriptional profiling of $\mathrm{CD} 4^{+}$hematopoietic progenitors from elderly humans. (A) Heatmap representation for the transcription of genes that are differentially expressed in HPCs from old $(0, n=8)$ healthy adults compared with young $(Y, n=4)$ or middle-aged (M, $n=7)$ subjects. The expression of selected genes in CD34+CD45 10 Lin- cells, FACS isolated ex vivo from donor PBMCs, was assessed using a Fluidigm microfluidics system. (B) Network diagram of genes differentially expressed in elderly HPCs with corresponding pathways highlighted using Ingenuity Pathway Analysis. The red or green colors indicate the degree of upregulation or downregulation in gene expression compared with housekeeping gene expression.

Altered transcriptional profile of hematopoietic progenitors from the elderly. Under steady-state conditions, HSCs are largely quiescent and undergo slow self-renewal (25). However, murine studies suggest that in response to stress during the course of aging and modifications of the environment, HSCs exit quiescence, enter cell cycling, and differentiate (2). To further characterize HPCs from aged humans, we next performed gene expression profiling of purified circulating $\mathrm{CD} 34^{+}$cells. Based on a hypothesis-driven approach, we assessed the expression of a selection of 80 genes associated with cell cycle, tumor suppressor pathways, nucleotide excision repair, telomere maintenance, or lineage differentiation (Supplemental Table 1) using a multiplex real-time PCR approach adapted to the study of rare CD $34^{+} \operatorname{Lin}^{-} \mathrm{CD} 45^{\mathrm{dim}}$ HPCs FACS isolated from elderly blood samples. Transcriptional analyses revealed differential clusters of expression between HPCs from aged individuals and HPCs from younger subjects (Supplemental Figure 2). In particular, the expression of a set of genes was significantly increased in elderly HPCs, suggesting an active, rather than quiescent, state of old HPCs (Figure 2A). We then performed a distinct network analysis based on the expression on this series of genes compared to housekeeping gene expression within old HPCs (Figure 2B and Supplemental Table 2). This analysis highlighted a number of pathways potentially altered in HPCs from aged individuals. These included the NF- $\mathrm{B}$, along with the mTOR and Wnt pathways, suggesting increased mobilization and cell cycling in these cells. Of note, the tumor suppressor- and senescence-related p53 pathway appeared to be also induced, which may influence HPC proliferation and survival. This altered gene expression profile prompted us to investigate in more detail the highlighted pathways to better understand the physiological alterations of old HPCs.

Increased mTOR pathway activity and mitochondrial stress in old HPCs. MTOR is a key regulator of the metabolic activity of the cells and is known to stimulate cell cycling, mitochondrial mass and activity, while inhibiting 
A

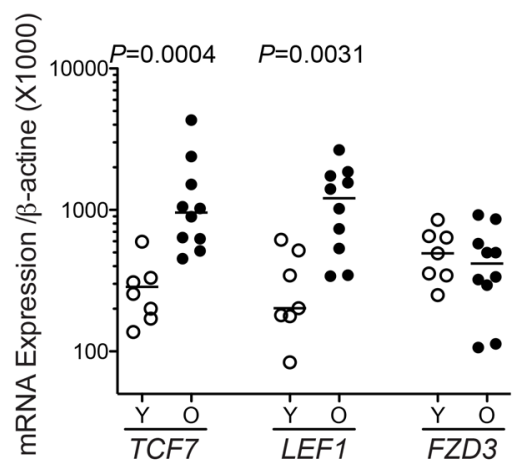

C

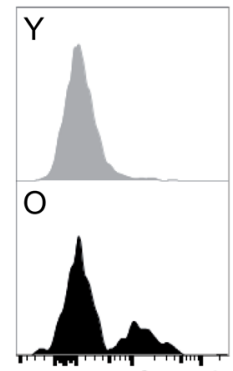

Phospho S6K

E

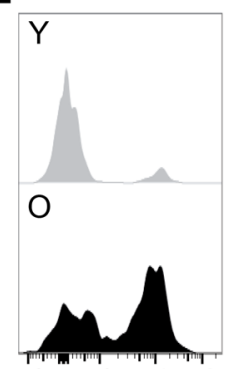

CellRox

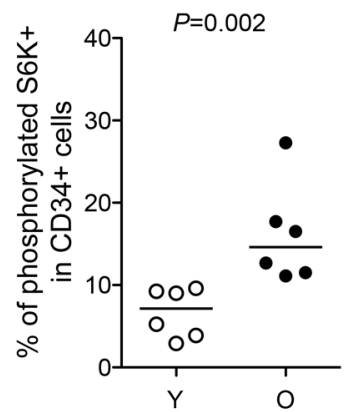

B

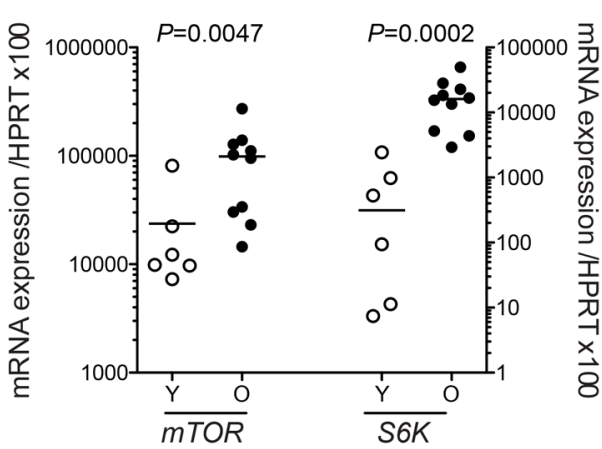

D
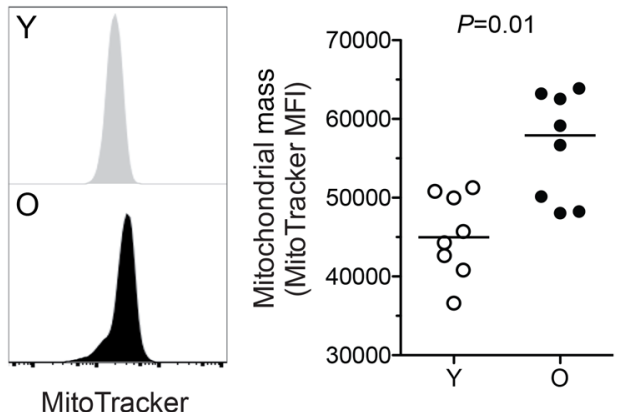

$\mathbf{F}$

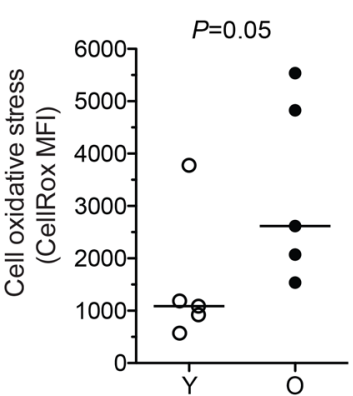

$\mathbf{F}$

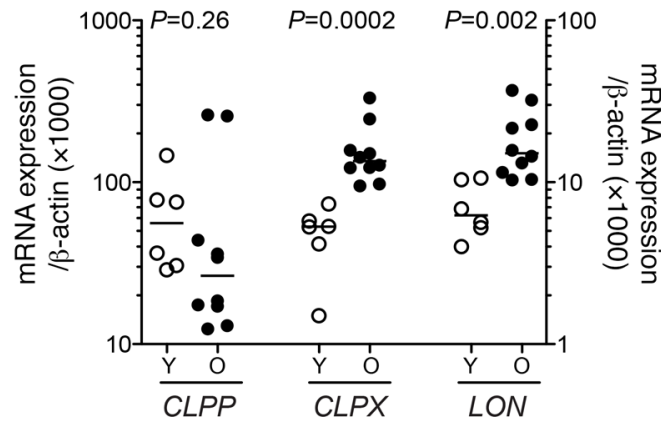

Figure 3. Upregulated mTOR pathway and mitochondrial stress in old hematopoietic progenitors. (A) mRNA expression levels of the Wnt pathwayrelated genes TCF7, LEF1, and FZD3 in FACS-isolated HPCs from young $(\mathrm{Y}, n=7)$ or old $(0, n=10)$ subjects. (B) mRNA expression levels of the mTOR pathwayrelated genes $M T O R$ and S6K in FACS-isolated HPCs from young $(n=6)$ or old $(n=10)$ subjects.

(C) Representative staining and median fluorescence intensity (MFI) levels for phosphorylated S6K in bead-enriched CD34+ cells from young or old adult PBMCs.

(D and E) Representative staining and MFI levels of MitoTracker (mitochondrial mass marker) and CellRox (cellular ROS marker) in bead-enriched $\mathrm{CD}_{3} 4^{+}$cells from young or old adult PBMCs. (F and G) mRNA expression levels of genes associated with mitochondrial protein folding stress (CLPP, CLPX, and $L O N$ ) and mitochondrial stress response (HSP6O, HSP7O) in FACS-isolated HPCs from young $(n=6)$ or old $(n=9)$ subjects. (H) Frequency of young or old CD34 ${ }^{+}$cells expressing the nuclear antigen Ki67. (I) Representative staining for $\mathrm{Ki} 67$ in $\mathrm{CD}^{+}$ or $\mathrm{CD}_{3} 4^{+}$cells from an elderly individual, highlighting differential Ki67 expression levels. The Mann-Whitney test was used for comparisons. Bars indicate the median.
G

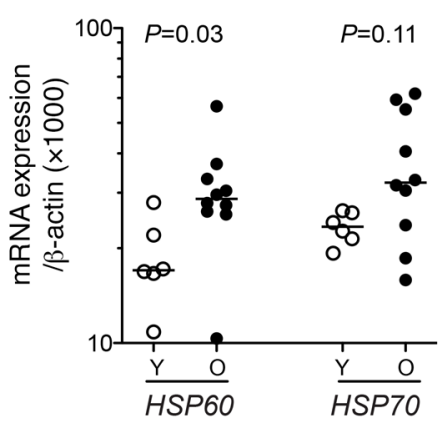

H

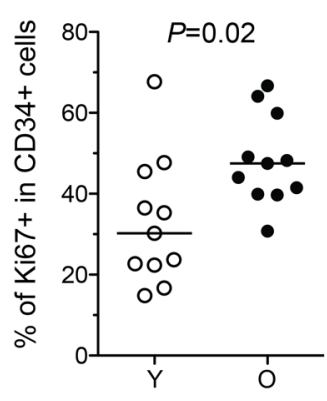

I

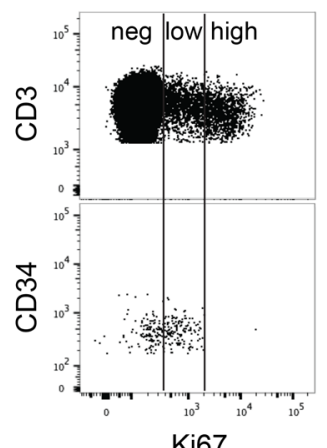

autophagy (26). To confirm the active state of old HPCs, we assessed several parameters related to the mTOR pathway. We first found in old HPCs the upregulated expression of the genes TCF7 and LEF1, which encodes factors belonging to the Wnt complex, an early actor of the mTORC1 pathway (Figure 3A). In contrast, the expression of $F Z D 3$, which encodes a negative regulator of the Wnt pathway, was not altered. Increased basal activity of the mTOR pathway in old HPCs was directly evidenced through increased transcript levels of $m T O R$ and $S 6 K$ (Figure 3B), as well as phosphorylation of the S6 kinase (S6K), which is downstream of mTORC1 signaling (Figure 3C). We did not observe evidence of increased glucose uptake or reduced autophagy levels 
A

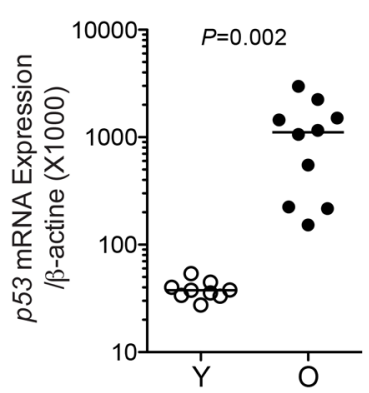

B

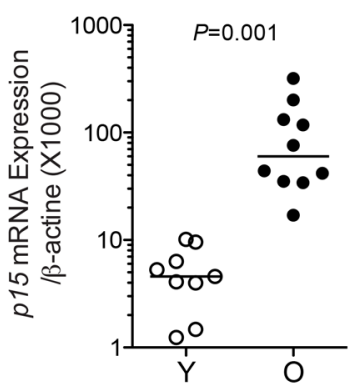

C

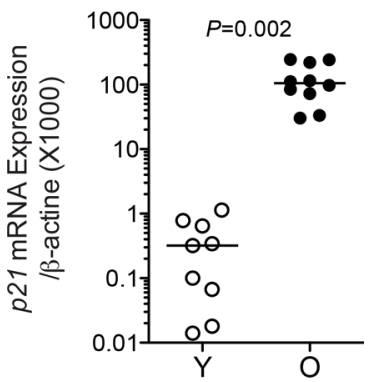

D

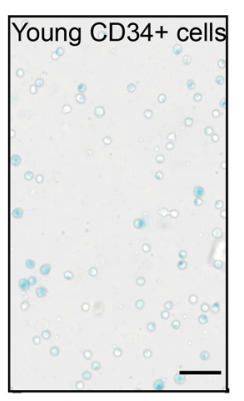

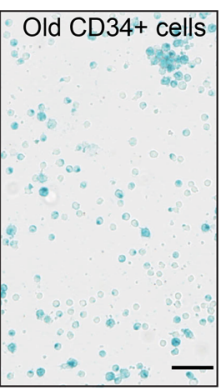
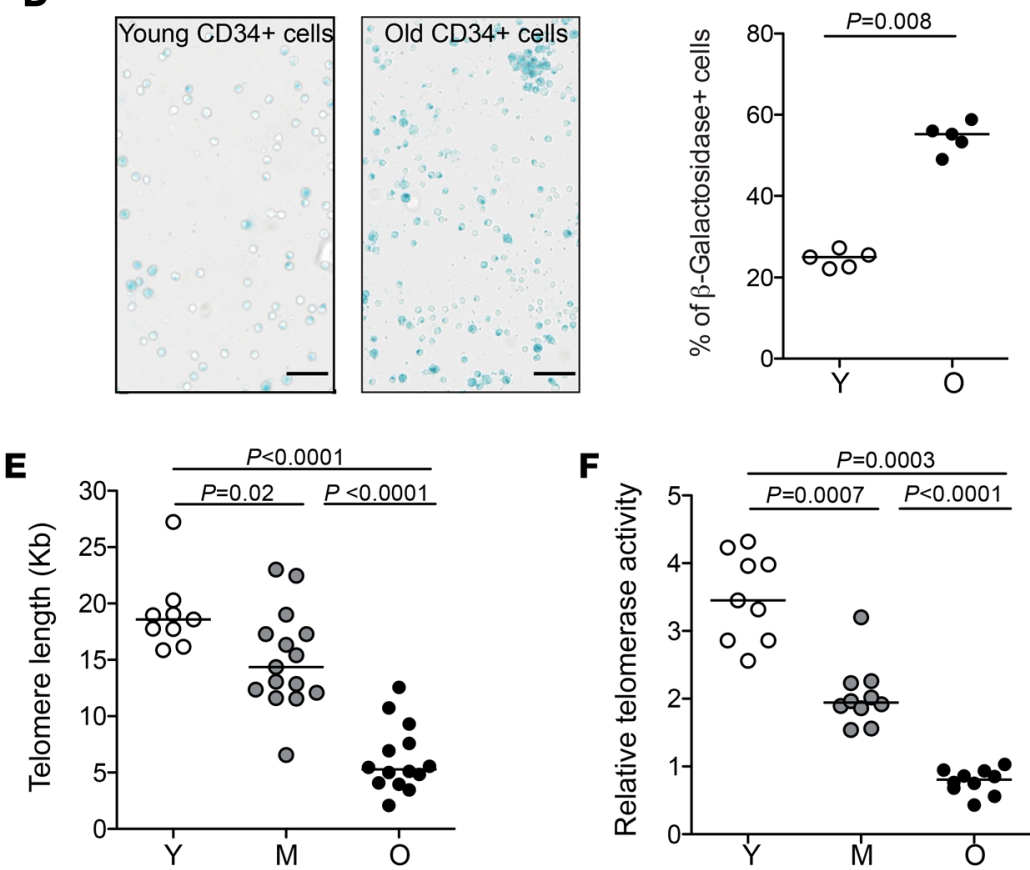

Figure 4. Increased markers of senescence in elderly hematopoietic progenitors. (A-C) mRNA expression levels of the cellular senescence pathway-associated genes $p 53, p 15$, and p21 in FACS-isolated HPCs from young $(\mathrm{Y}, n=9)$ or old $(0, n=10)$ individuals. (D) Representative $\beta$-galactosidase staining (left) and frequency of $\beta$-galactosidase-positive cells (right) within FACS-isolated CD34+ cells from old $(n=5)$ or young $(n=5)$ individuals. Scale bars: $50 \mu \mathrm{m}$. (E) Telomere length in CD34+ HPCs from young $(n=9)$, middle-aged $(\mathrm{M}, n=15)$, or old $(n=14)$ healthy adults. Telomere length was measured by qPCR in CD34+CD45 ${ }^{10} \mathrm{Lin}^{-}$cells FACS isolated from PBMCs, and Kb calculated based on a reference cell line. (F) Relative telomerase activity in $\mathrm{CD} 34^{+} \mathrm{HPC}$ from young, middle-aged, or old healthy adults. Telomerase activity was assessed using a TRAP assay in $\mathrm{CD} 34^{+} \mathrm{CD} 45^{10} \mathrm{Lin}^{-}$ cells FACS isolated from PBMCs. The MannWhitney test was used for comparisons. Bars indicate the median.

in old compared with young HPCs (Supplemental Figure 3, A and B). However, CD 34+ cells from the elderly showed increased markers of mitochondrial activity and stress. Indeed, mitochondrial mass and cell oxidative stress (Figure 3, D and E), as well as transcriptional levels of several factors related to the mitochondrial stress response (i.e., CLPX, LON, and HSP60) were elevated in these cells (Figure 3, F and G). Increased mTOR and mitochondrial activity are indicative of an active state and oxidative phosphorylation in old CD $34^{+}$cells. Old HPCs also presented a higher expression of the cell cycling entry marker Ki67, compared with young HPCs (Figure 3H). However, Ki67 expression in old HPCs showed a consistently low mean fluorescence intensity (MFI) (Figure 3I), indicative of cells in early cycle phases. This is in line with increased expression of PCNA, $C D K 6$, and $C D K 7$, which are also indicators of early cell cycle phases such as G1 and in particular S (Figure 2A). Altogether, these findings depict old HPCs as metabolically active and prone to enter cell cycle, but presenting mitochondrial stress and being mainly at early cell cycle stages.

Markers of cellular senescence in elderly HPCs. In addition to the higher transcriptional p53 levels in old HPCs, which we confirmed using classical quantitative PCR (qPCR) assay (Figure 4A), we assessed a series of senescence markers to better characterize the state of $\mathrm{CD} 34^{+}$cells from the elderly. While $p 16$ transcription was too low to be detected, these cells presented high expression levels of $p 15$ (CDKN2A) and $p 21$ (CDKN1A) (Figure $4, \mathrm{~B}$ and $\mathrm{C}$, respectively), which play key roles in cellular senescence programs and growth arrest. Staining for $\beta$-galactosidase, a classical marker of cellular senescence, was also prominent in old HPCs compared with young HPCs (Figure 4D). Another key marker associated with senescence is usually the length of telomeres, which are hexanucleotide (TTAGGG) tandem repeats of DNA at chromosome termini. Telomere attrition of 50 to $150 \mathrm{bp}$ occurs every cell division, although telomerase, a ribonucleoprotein enzyme complex, acts to limit this process by synthesizing telomeres. We thus assessed the possibility that telomere attrition occurs in aged human HPCs, 
A
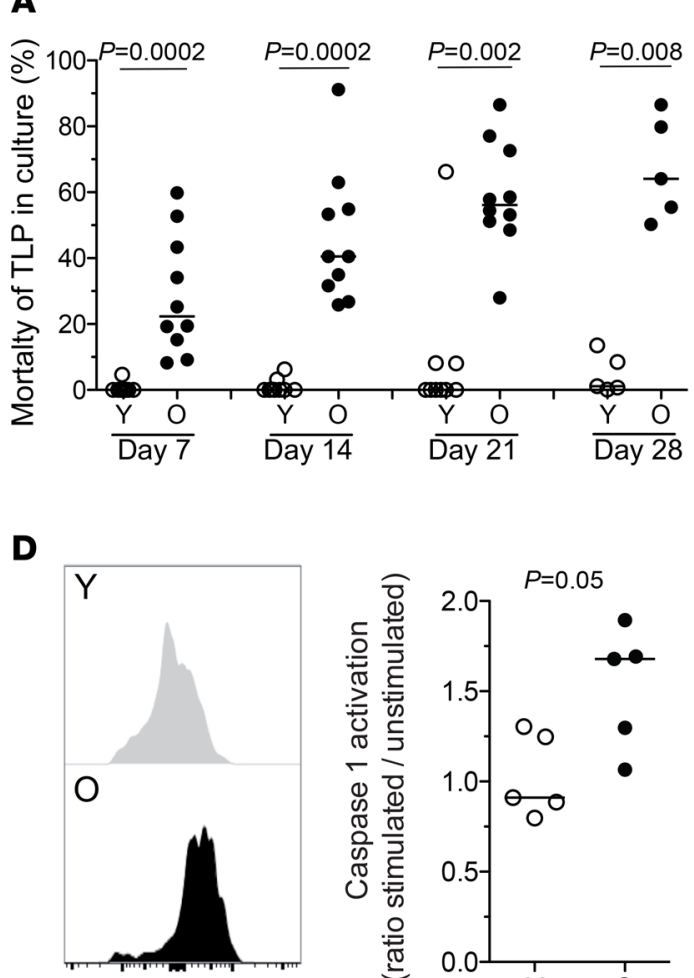

Cleaved caspase 1
B

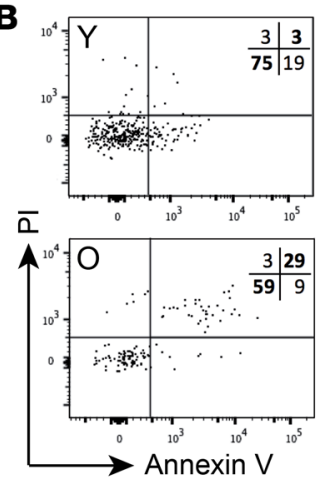

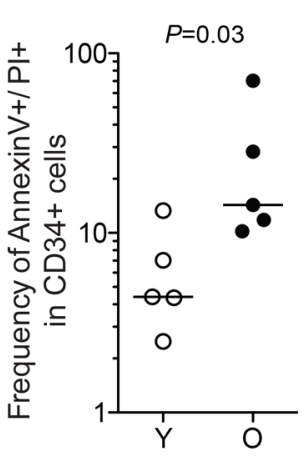

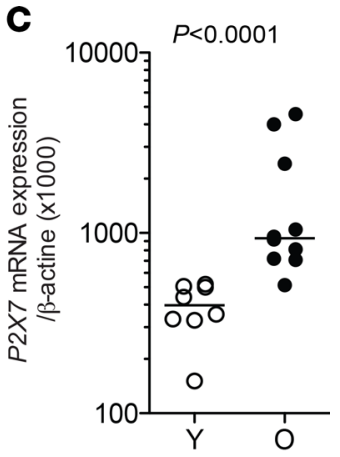

Figure 5. Increased cell death and pyroptosis in old hematopoietic progenitors. (A) Frequency of dead cells among TLPs (using LIVE/DEAD staining assay) at $7,14,21$, and 28 days upon in vitro differentiation of FACS-isolated CD34+ HPCs from young (Y, $n=9)$ or old ( $0, n=10)$ healthy adults in the OP9-DL1 coculture system. (B) Representative image and staining levels for annexin $V$ and PI in bead-enriched CD34+ cells from young ( $n=5$ ) or old adult $(n=5)$ PBMCs. (C) Increased mRNA expression of the proapoptotic gene $P 2 X 7$ in FACS-isolated HPCs from old ( $n=10$ ) healthy adults compared with HPCs from young $(n=7)$ subjects. (D) Representative staining and expression levels of cleaved caspase 1 in CD34 ${ }^{+}$cells from young ( $n=5$ ) or old $(n=5)$ adult PBMCs. Data are expressed as ratio of stimulated versus unstimulated cells. Bars indicate the median. (E) Enhancement of in vitro survival of TLPs at 14 or 21 days in the presence of PPAD (P2X7 inhibitor, at $20 \mu \mathrm{M}$ ) or VX-765 (caspase 1 inhibitor, at $0.1 \mu \mathrm{M})$ from day 7 in the $0 P 9-D L 1$ culture system. Survival is expressed as the ratio of dying cells at day 14 or 21 over day 7 in culture ( $n=5$ in the presence of PPAD or VX-765 and $n=10$ in the absence of inhibitors). Columns indicate mean (+SEM). The Mann-Whitney test was used for comparisons.

in particular since to our knowledge this issue has never been directly addressed in humans. To this end, we established qPCR-based methods to measure telomere length, as well as telomerase activity, in low cell numbers, adapted to the study of FACS-isolated human HPCs. This approach revealed that HPCs from elderly adults present significant shortening of their telomeres, with lengths approaching those found in senescent cells (Figure 4E and Supplemental Figure 4A). Over the different age groups, there was a good correlation between age of the donor and HPC telomere length (Supplemental Figure 4B). We then assessed telomerase activity in HPCs according to the donor age. HPCs from elderly adults displayed particularly reduced telomerase activity (Figure 4F). Telomere length and telomerase activity were in general well correlated in HPCs across the age spectrum (Supplemental Figure 4C). Altogether, these findings show that HPCs from aged individuals approach a state of senescence.

Pyroptosis-associated cell death in old HPCs. In culture, old-HPC-derived TLP numbers reached a plateau quickly, suggesting a weaker expansion or survival capacity, compared with young-HPC-derived TLPs (Figure 1G). The latter was supported by evidence of increased mortality of TLPs derived from elderly HPCs, early in culture, in clear contrast with TLPs derived from young-subject HPCs (Figure 5A). Increased cellular activity, but poor survival in vitro, may reflect an increased susceptibility to cell death of old HPCs. We therefore assessed cell death markers on circulating CD $34^{+}$cells directly ex vivo. Old HPCs displayed a higher frequency of cells positive for both annexin V and propidium iodide (PI) (Figure 5B and Supplemental Figure 5). In the absence of increased annexin V staining only, this can be characteristic of pyroptosis (27). Pyroptosis is an inflammatory form of programmed cell death, which is induced through the activation and cleavage of caspase 1 upon signaling through the purinergic receptor P2X7 (28). In line with this observation, we found 
A
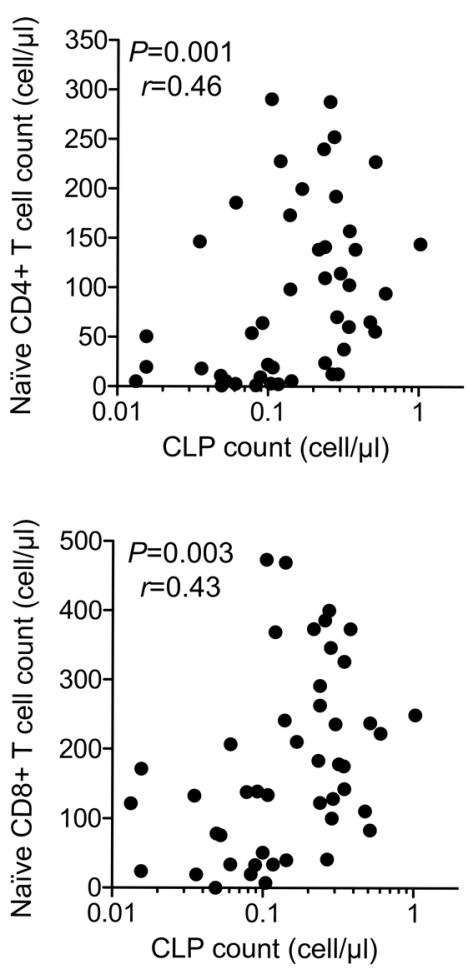

B
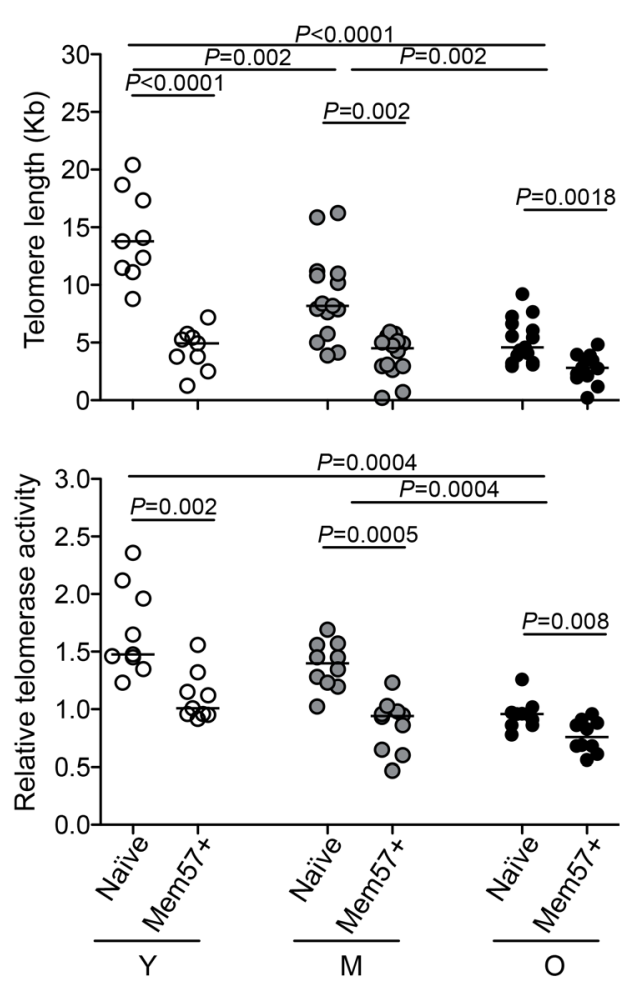

CD8+ T cells
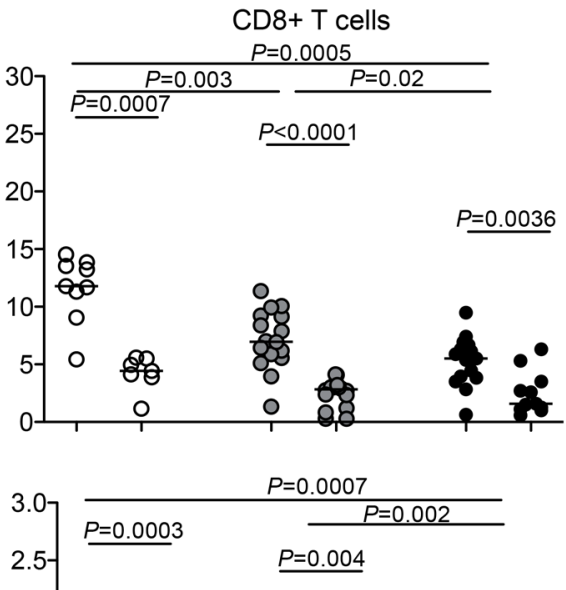

3.07 $1.5 \frac{P=0.0007}{P=0.0003} \quad \frac{P=0.002}{P=0.004}$
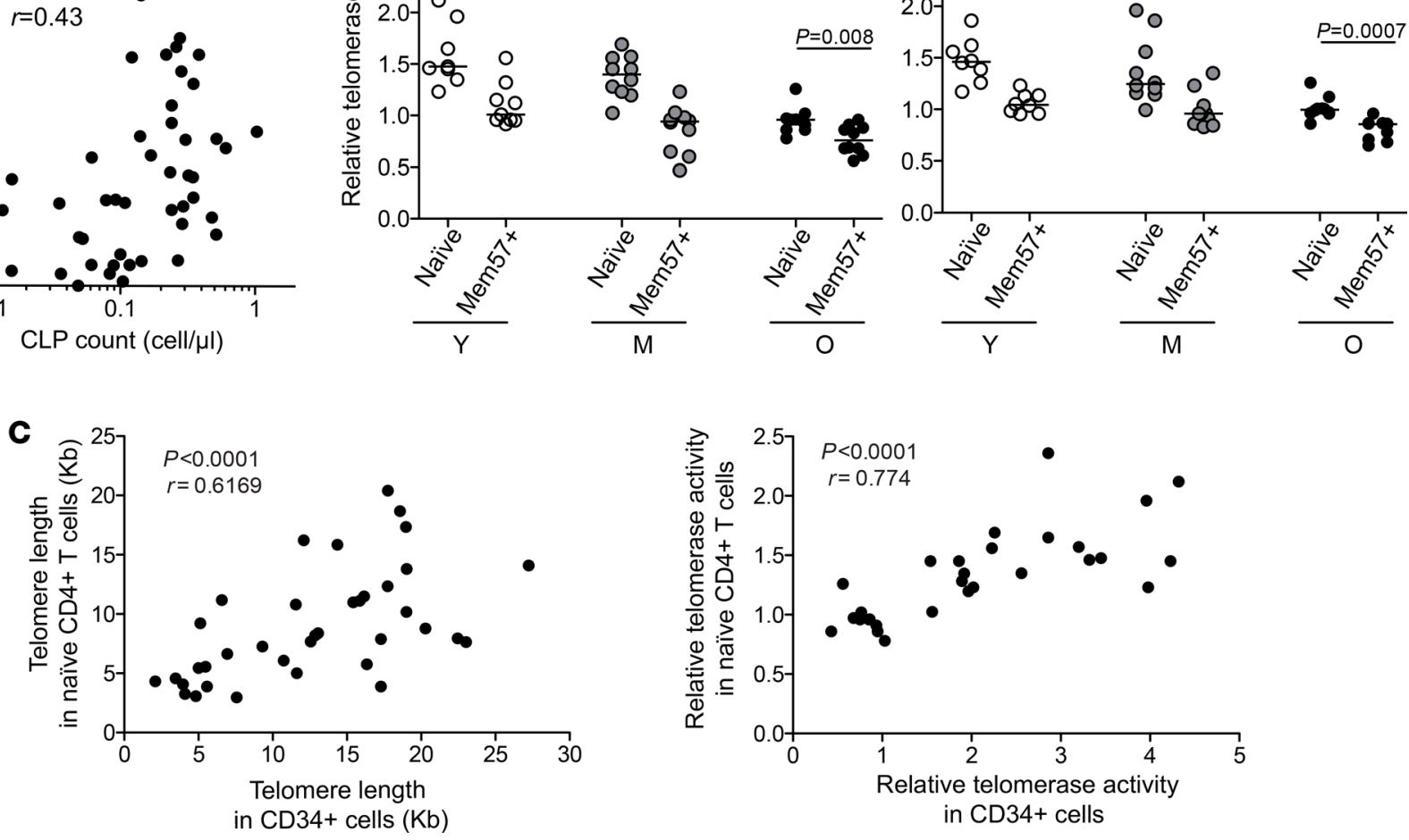

Figure 6. Associations between circulating HPCs and naive $\mathbf{C D 4}^{+}$or $\mathbf{C D 8}{ }^{+} \mathbf{T}$ cell properties. (A) Correlations between $\mathrm{CLP}$ and naive $\mathrm{CD} 4^{+}$or $\mathrm{CD} 8^{+} \mathrm{T}$ cell ex vivo absolute counts. (B) Telomere length (top panels) and relative telomerase activity (bottom panels) in naive or senescent CD57+ memory CD4+ or CD8 ${ }^{+}$T cells FACS isolated from young $(Y, n=9)$, middle-aged $(M, n=15)$, or old $(0, n=14)$ healthy adult PBMCs. (C) Correlations between CLP and naive $\mathrm{CD}^{+}$or $\mathrm{CD}^{+} \mathrm{T}$ cell telomere length or telomerase activity. The Mann-Whitney test was used for comparisons. Bars indicate the median. Spearman's rank test was used to determine correlations.

an overexpression of P2X7 mRNA levels in elderly HPCs (Figure 5C). Upon stimulation, old HPCs also displayed increased caspase 1 activation compared with young HPCs (Figure 5D), thus highlighting the high susceptibility of these cells to cell death via pyroptosis. In order to confirm the role of pyroptosis, we tested the influence of inhibitors of P2X7 (pyridoxal-phosphate-6-azophenyl-2', 4'-disulfonate [PPAD]) or caspase 1 (VX-765) on the capacity of old HPCs to survive and differentiate into T lymphocytes in vitro. While it had no effect on young cells, the presence of either PPAD or VX-765 in OP9-DL1 coculture assays significantly improved old TLP survival in vitro (Figure 5E). There was nonetheless no improvement in TLP production and differentiation (data not shown). Although inhibitors of pyroptosis may not improve lymphopoiesis directly, they may help to rescue old $\mathrm{CD} 34^{+}$cells from cell death.

Impact of old HPC alterations on the naive T cell compartment. Since HPCs are upstream elements of lymphopoiesis, old-age-related alterations of the compartment are likely to have a direct impact on the 
generation and properties of new lymphocytes. Interestingly, we found that circulating CLP numbers correlated with the counts of naive $\mathrm{CD}^{+}$or $\mathrm{CD}^{+} \mathrm{T}$ cells (Figure $6 \mathrm{~A}$ ), supporting a direct link between these 2 compartments. Moreover, telomere length as well as telomerase activity in mature naive $\mathrm{CD} 4^{+}$or $\mathrm{CD} 8^{+}$ $\mathrm{T}$ cells were consistent with those of the $\mathrm{CD} 34^{+}$precursors (Figure $6 \mathrm{~B}$ and Supplemental Figure 4D). Naive $\mathrm{CD}^{+}$or $\mathrm{CD}^{+} \mathrm{T}$ cells from aged individuals thus displayed short telomere length along with very low telomerase activity, compared with younger counterparts. Although increased homeostatic proliferation of naive T cells in the elderly (29) might by itself impact telomere length in this population, a direct imprinting of HPC properties on the naive T cells was supported by a strong correlation between naive $\mathrm{T}$ cell and CD34+ HPC telomere length or telomerase activity across age groups (Figure 6C). The proliferative potential of the naive T cells may therefore be directly limited by the state of the HPCs at an advanced age. Strikingly, the attrition of old naive $\mathrm{CD}^{+}$or $\mathrm{CD}^{+} \mathrm{T}$ cell telomeres and their telomerase activity reached levels close to those observed in memory $\mathrm{CD} 4^{+}$or $\mathrm{CD} 8^{+} \mathrm{T}$ cells with a $\mathrm{CD} 28^{-} \mathrm{CD} 57^{+}$phenotype (Figure 6, B and C), commonly considered as close to senescent cells (30). Overall, these data suggest that age-related disruptions of the HPC compartment, including the attrition of their telomeres and the dampened telomerase activity are likely to affect lymphopoiesis in general, from the HPC self-renewal capacity to the production of robust and long-lived immunocompetent cells like naive T lymphocytes in the elderly.

\section{Discussion}

The replacement of old and worn out immunocompetent cells with aging relies on the primary hematopoietic compartments of HSCs and HPCs. However, this replenishment may be limited due to functional impairments of these cells in aged individuals $(1,2)$. By highlighting a preferential deficiency of the lymphopoietic potential of old $\mathrm{CD} 34^{+}$cells, the phenotypic and functional properties of elderly circulating HPCs confirmed findings obtained in mice or in humans using HSCs from bone marrow, and therefore validate the study of circulating HPCs as an informative option to characterize the disruption of hematopoietic capacity in elderly humans. The present study of HPCs provides potentially new insights underlying the altered state of these cells with advanced age in humans.

In line with knowledge established in mice, HPCs in the elderly, rather than being quiescent, are in an active state, which is a likely consequence of the strong proinflammatory environment characteristic of advanced age and their mobilization to replenish or replace declining immunocompetent cell compartments. Old HPCs indeed present evidence of active metabolism and cell cycle entry, along with a predisposition to further lymphoid differentiation, as observed in in vitro culture. Our findings draw particular attention to the active Wnt and mTOR pathways in these cells. A heightened mTOR pathway has been associated with intense mitochondrial activity (31), which emerges as traits of old CD $34^{+}$cells in our study. HPCs from elderly people indeed displayed increased mitochondrial mass and levels of mRNA encoding mitochondrial stress-related factors, indicative of oxidative phosphorylation.

Paradoxically, CD34+ cells also display evidence of cellular stress, such as ROS production, and senescence with advanced age. We report here deficient telomerase activity in old human $\mathrm{CD} 34^{+}$cells together with significant attrition of telomeres, to levels approaching those of cells considered senescent (i.e., less than $5 \mathrm{kbp}$ ). This is consistent with earlier work showing telomere shortening in elderly peoples' neutrophils, short-lived cells that require continuous renewal from HPCs (32). HSC functional deficiency related to telomere attrition is also in line with observations in humans with short telomeres (due to congenital telomerase deficiencies) who develop bone marrow failure (33-35). Telomere length is a record of the cell proliferative history, such that the erosion of HPC telomeres is most likely a consequence of excessive self-renewing and cell cycling during the course of physiological aging. Moreover, we observed in old HPCs increased expression levels of p53, p21, and p15, which are cell-senescence-associated factors. Strong telomere erosion, but also ROS production, and potentially accumulation of DNA damage (15) can result in the induction of these factors and a cellular senescence program in old HPCs. These factors are known to block cell cycling at early G1 and S stages, which characterize old HPCs. Of note, the activation of mTORC1 and S6 kinases has also been recently shown to play an important role in the induction of cell senescence (36).

The activation of HPCs that display senescence features likely leads to the induction of increased cell death in these cells. Our analyses point towards an increase of pyroptosis in old HPCs, which present a high frequency of annexin V and PI double-positive cells, together with elevated levels of $P 2 X 7$ transcripts and of cleaved caspase 1 upon activation. Pyroptosis is known to occur upon NF- $\kappa B$ pathway induction, together with signaling through P2X7, upon extracellular ATP binding. These are features of old CD34 
cells, as observed in the present study, and can result from proinflammatory signals and excessive ATP production through oxidative phosphorylation in these cells, respectively. Of note, these findings are in line with a recent report showing high levels of mRNA encoding for P2X7 in HPCs from HIV-1-infected patients (28), another setting with elevated systemic inflammation and exhausted lymphopoiesis (37). Altogether, these findings offer a new understanding in the impairment mechanisms occurring in elderly human HPCs, and leading to their decreased regenerative, differentiation, and survival capacity. Our study demonstrates that several active pathways collide in old HPCs, and draws a picture of these cells as being overly mobilized, but functionally biased and exhausted, approaching senescence and prone to cell death. Future studies will aim at dissecting the properties of the different subpopulations composing the pool of human $\mathrm{CD}_{3} 4^{+}$cells in the circulation or the bone marrow, in order to determine if the alterations described in the present study apply to all cells. Distinct subpopulations, such as primitive HSCs, CLPs, or CMPs may indeed display specific impairments, or may have preserved functions, as recently shown in mice (10).

Beyond the characterization of age-related HPC alterations, it is also important to understand the impact on progeny cells. The correlation between circulating CLP and naive T cell numbers suggests a link between altered HPC attributes and the production of new mature cells. It is also tempting to speculate that functional alterations of HPCs or HSCs are transmitted to mature immunocompetent cells. For instance, DUSP6 (dual specificity phosphatase 6), whose expression is higher in blood HPCs from elderly individuals compared with young adults, as well as in elderly human bone marrow HSCs (13), was identified as a factor responsible for the reduced responsiveness and proliferative capacity of naive $\mathrm{CD} 4^{+} \mathrm{T}$ cells from elderly people (38). Most importantly, short telomere lengths and telomerase activity deficiency may be directly passed from HPCs to naive T cells in the elderly. This implies that new mature cells start off with a significantly reduced proliferative potential, thus limiting their expansion capacity upon antigen-driven stimulation and the efficacy of the subsequent immune response. Short telomere length, deficient telomerase activity, and reduced proliferative capacity may partially explain the reduced $\mathrm{T}$ cell priming efficacy reported in elderly humans (39). Along the same line, telomerase activity has been associated with the lifespan of human $\mathrm{T}$ lymphocytes (40), and long telomere length with a robust lymphocyte response to influenza vaccine in elderly adults (41). Therefore, significant telomere attrition in HPCs and HSCs of elderly humans is likely to have a direct consequence for immune competence with aging.

Rejuvenating old HSCs to boost their hematopoietic capacity and ultimately rescue the number and functionality of immunocompetent cells like $\mathrm{T}$ lymphocytes is a major research goal of the aging field. By highlighting different altered pathways (i.e., cell death, metabolism, cell senescence) in old HPCs, our present work identifies several potential strategies to target in order to reach this goal. Current data support the idea that rejuvenation of aged HSCs may be achieved using pharmacological inhibitors. In this context, improving HSC survival by blocking pyroptosis may prove to be useful, as shown in our in vitro assays, although it had only a limited impact on improving TLP differentiation. A persistent active metabolic state in HPCs likely also represents an important functional bias in terms of defective lymphopoiesis with old age. Increased activation of Wnt and mTOR pathways has been shown to associate with exhaustion of stem cells after serial transplantation in mice (42). Interestingly, targeting the mTOR pathway (in particular mTORC1) is showing increasing interest and potential. In aged mice, rapamycin treatment was shown to restore HSC self-renewal and reconstitution potential, and improve influenza virus vaccine responsiveness (43). However, although promising, these pharmacological approaches may fail to rescue HSC replicative capacity and effective lymphopoiesis in elderly humans, as deficits may be primarily limited by the cellular senescence program induced in these cells. The weak telomerase activity and substantial shortening of telomeres measured in old HPCs, and activation of a DNA-damage response, may compromise HPC replicative capacity. Reverting stem cell exhaustion will likely require strategies to enhance telomerase activity specifically in HSCs or to use cellular reprogramming approaches, like induced pluripotent stem cell technology (44). Alternatively, prevention strategies at earlier ages to preserve telomere length and replicative potential of HSCs with aging may be necessary.

\section{Methods}

Study patients. Three groups of volunteers of European descent were enrolled in this study: (a) young (18 $<$ age $<25$ ), (b) middle-aged ( $30<$ age $<55)$, and (c) elderly ( $>70$ years old) adults. All individuals were healthy. Individuals with malignancies, acute diseases, or advanced stages of severe chronic diseases, such as chronic inflammatory disease, atherosclerotic disease, congestive heart failure, poorly controlled diabetes mellitus, renal or hepatic disease, or chronic obstructive pulmonary disease, as well as individuals 
under immunosuppressive therapy were excluded from the study. Venous blood samples were drawn into anticoagulant tubes and peripheral blood mononuclear cells (PBMCs) were isolated by density gradient centrifugation according to standard protocols.

Flow cytometry. Directly conjugated antibodies were obtained from the following vendors: CD1a (APC, 559775), CD4 (HV500, 560768), CD3 (FITC, 555916), CD8 (BV650, 563822), CD38 (APC, 345807), CD34 (PE, 345802), CD45RA (V450, 560362), and lineage cocktail (CD3, CD14, CD16, CD19, CD20, CD56; FITC, 340546) from BD Biosciences; CD45RA (ECD, IM2711U), CD5 (APCAF700, A78836), and CD117 (PE-Cy7, B49221) from Beckman Coulter; CD27 (Alexa Fluor 700, 302814), CD10 (APC-Cy7, 312212) and CD57 (PB, 322316) from BioLegend; and CD90 (PerCP Cy5.5, 45-0909-42) and CD7 (Alexa Fluor 700, 56-0079-42) from eBioscience. Cell surface staining was performed using standard methodologies. Flow cytometry-based assessments of HPC properties were performed on magnetic bead-enriched $\mathrm{CD}_{3} 4^{+}$cells. For ROS and mitochondrial mass analyses, $\mathrm{CD} 4^{+}$cells were washed with Dulbecco's PBS (DPBS), incubated for 30 minutes at $37^{\circ} \mathrm{C}$ with CellROX Green Reagent ( $5 \mu \mathrm{M}$; Invitrogen, C10444), or Mitotracker Red FM (500 nM; Thermo Fisher Scientific, M22425) followed by surface staining for 15 minutes at room temperature in DPBS. For autophagy analysis, CD $34^{+}$cells were incubated with Cyto-ID (1:1,000; Enzo, ENZ-51031-0050) in staining media. Cells were then washed with staining media followed by surface staining for 15 minutes at room temperature. For intracellular phospho-S6K staining, CD34 ${ }^{+}$cells were washed in PBS, fixed with BD Cytofix (BD Biosciences, 554655) for 10 minutes at $37^{\circ} \mathrm{C}$, and permeabilized in BD Perm Buffer III (BD Biosciences, 558050) for 30 minutes at $4^{\circ} \mathrm{C}$, washed and stained in BD Stain Buffer (BD Biosciences, 554656) with anti-phospho-S6 ribosomal protein monoclonal antibody (Cell Signaling Technology, 8520S) for detection of cell-surface-associated protein for 1 hour at room temperature. For intracellular caspase 1 staining, CD $34^{+}$cells were washed in PBS, fixed in Cytofix/Cytoperm buffer (BD Biosciences), for 20 minutes at $4^{\circ} \mathrm{C}$, washed, and stained in PermWash (BD Biosciences) with primary antibody rabbit anti-caspase 1 (Thermo Fisher Scientific, PA5-38099) for 30 minutes at $4^{\circ} \mathrm{C}$. Cells were then washed in PermWash and incubated with anti-rabbit AF-405 secondary antibody (Invitrogen, A31556) for 20 minutes at $4^{\circ} \mathrm{C}$. Cells were washed once more with PermWash and resuspended in PermWash for analyses. For intracellular Ki67 staining, $\mathrm{CD} 34^{+}$cells were first stained with surface markers in PBS and then permeabilized with a Perm/Fix kit (eBioscience, 00-5523-00) before the addition of Ki67 FITC antibody (BD Pharmingen, 556026). For measurement of glucose uptake, CD34 cells were washed in PBS and incubated in $100 \mu \mathrm{M}$ 2-(n-(7-nitrobenz-2-oxa-1,3-diazol-4-yl)amino)-2-deoxyglucose (2-NBDG; Invitrogen, N13195) for 20 minutes at $37^{\circ} \mathrm{C}$. Cells were then washed with cold PBS and then stained with surface markers. To assess cell death, staining with annexin V FITC and PI (Thermo Fisher Scientific) was evaluated by flow cytometry according to the manufacturer's instructions. This assay enabled the identification of early apoptotic cells (annexin $\mathrm{V}^{+} / \mathrm{PI}^{-}$), late apoptotic/ pyroptotic cells (annexin $\mathrm{V}^{+} / \mathrm{PI}^{+}$), and primary necrotic cells (annexin $\mathrm{V}^{-} / \mathrm{PI}^{+}$) from live cells (annexin $\mathrm{V}^{-} / \mathrm{PI}^{-}$). All staining was analyzed on an LSRFortessa flow cytometer (Becton Dickinson) and data analyzed using FlowJo v10.6 (Tree Star, Inc).

In vitro OP9-DL1 coculture assays. CLP potential was established in vitro by culturing peripheral sorted CD34+ HPCs on an OP9 human delta-1 stromal cell line (OP9-DL1), obtained from ATCC (CRL2749, LGC Promochem). CLP potential was assayed by plating approximately 5,000 FACS-isolated $\mathrm{CD} 34^{+} \mathrm{CD} 45^{\mathrm{lo}} \mathrm{Lin}^{-}$cells per well of a 24-well plate previously coated with OP9-DL1 cells (using 20,000 cells the day before the coculture). The coculture was conducted in $\alpha$-MEM (Invitrogen) freshly reconstituted with Milli-Q water and supplemented with 20\% Defined Fetal Bovine Serum (Hyclone, Thermo Fisher Scientific) and the following cytokines: $2 \mathrm{ng} / \mathrm{ml} \mathrm{IL-7,} 5 \mathrm{ng} / \mathrm{ml} \mathrm{FLT-3,} 10 \mathrm{ng} / \mathrm{ml} \mathrm{SCF}$, and $10 \mathrm{ng} /$ $\mathrm{ml}$ TPO (R\&D Systems). The stroma was replaced weekly. The P2X7 inhibitor PPAD (Sigma-Aldrich, 20 $\mu \mathrm{M}$ ) or the caspase 1 inhibitor VX-765 (Sigma-Aldrich, $0.1 \mathrm{mM}$ ) was added at day 0 or 7 of the culture as indicated. For the first 4 weeks of culture, flow cytometry was performed weekly to detect CLP induction $\left(\mathrm{CD} 34^{+} \mathrm{CD} 45 \mathrm{RA}^{+} \mathrm{CD}^{+}\right)$, after exclusion of dead cells using a LIVE/DEAD Fixable Violet Dead Cell Stain Kit (Life Technologies). Early T cell differentiation was monitored through analysis of the expression of CD5 and CD1a within CLPs.

Gene expression analysis. Quantification of gene expression was performed using either the Fluidigm BioMark platform as described previously (45) or classical reverse transcription qPCR (RT-qPCR). Viable CD $34^{+} \mathrm{CD} 45^{10} \mathrm{Lin}^{-}$cells were sorted by flow cytometry ( 25 cells/well) into duplicate assay wells 
containing the RT-PCR reaction and cDNA synthesis mix. Transcripts were amplified using the SuperScript III Platinum One-Step qRT-PCR system (Invitrogen), according to the manufacturer's instructions. The 2-way hierarchical clustering and network diagram analyses were performed using JMP (SAS), Qlucore Omics explorer, and Ingenuity Pathway Analysis (QIAGEN) software. For the gene expression quantification, data were analyzed with the $\Delta \Delta \mathrm{Ct}$ method and normalized to house keeping gene expression. Primers for RT-qPCR were designed using at least 1 exon junction-binding site per primer pair. The thermal cycling profile for amplification was $95^{\circ} \mathrm{C}$ for 10 minutes, followed by 40 cycles of $95^{\circ} \mathrm{C}$ for 15 seconds and $60^{\circ} \mathrm{C}$ for 1 minute. To ensure the amplification specificity, the melting curve program was set as follows: $95^{\circ} \mathrm{C}$ for 15 seconds, $60^{\circ} \mathrm{C}$ for 1 minute, and $95^{\circ} \mathrm{C}$ for 15 seconds, immediately after the PCR cycles. Information on the primers and probes used in this study is provided in Supplemental Table 3.

Senescence-associated $\beta$-galactosidase activity assay. Cytochemical staining for senescence-associated $\beta$-galactosidase was performed using a Senescence $\beta$-Galactosidase Staining Kit (Cell Signaling Technology, 9860) at pH 6. FACS-isolated CD34+ cells were washed with PBS and seeded (500 cells/well) in 24-well plates precoated with poly-L-lysine and fixed in $1 \mathrm{ml}$ of fixative per well for 30 minutes at $37^{\circ} \mathrm{C}$. The cells were washed with PBS and incubated overnight at $37^{\circ} \mathrm{C}$ without $\mathrm{CO}_{2}$ with $1 \mathrm{ml}$ /well of $\beta$-galactosidase substrate $\mathrm{X}-\mathrm{Gal}$. The senescent cells were observed under a microscope and counted using ImageJ software (NIH).

Telomere length measurement. Genomic DNA (gDNA) was extracted from sorted cells with the QIAmp DNA Mini kit (QIAGEN), following the manufacturer's instruction. Quantification of gDNA samples was performed using a NanoDrop 2000 (Thermo Fisher Scientific). Relative telomere length was measured as the ratio of standard DNA quantities for telomere template over single-copy gene 36B4 determined using qPCR, as described previously by Cawthon et al. with some modifications (46). Measurement was performed in triplicate. Telomere and 36B4 PCRs were done on separate plates. The intra-assay coefficient of variation was less than 5\%. PCR primers were (Tel-F, 5'-CGGTTTGTTTGG GTTTGGGTTTGGGTTTGGGTTTGGGTT-3' and Tel-R， 5'-GCTTGCCTTACCCTTACCCTTACCCTTACCCTTACCCT-3') for telomere PCR and (36B4-F, 5'-CAGCAAGTGGGAAGGTGTAATCC-3' and 36B4-R, 5'-CCCATTCTATCATCAACGGGTACAA-3') for the 36B4 PCR. The final primer concentration was $400 \mathrm{nM}$ for all primers. The PCR mix contained Power SYBR Green Master Mix (Life Technologies) and 2 ng of gDNA. qPCR was performed on a Bio-Rad 7300 Thermal cycler. The thermal cycling profile for telomere amplification consisted of a preparation step of 2 minutes at $50^{\circ} \mathrm{C}, 10$ minutes at $95^{\circ} \mathrm{C}$, followed by 35 cycles at $95^{\circ} \mathrm{C}$ for 15 seconds, $56^{\circ} \mathrm{C}$ for 2 minutes, and $72^{\circ} \mathrm{C}$ for 1 minute. The 36B 4 amplification profile was 2 minutes at $50^{\circ} \mathrm{C}, 10$ minutes at $95^{\circ} \mathrm{C}$, followed by 35 cycles of $95^{\circ} \mathrm{C}$ for 15 seconds and $58^{\circ} \mathrm{C}$ for 1 minute. To convert the ratio of starting quantity into telomere length in base pairs, a serial dilution of DNA samples from the 293T cell line (obtained from ATCC; ref. CRL-3216, LGC Promochem) was run simultaneously in classical Southern blot and qPCR.

Telomerase activity measurement. Relative telomerase activity (RTA) was measured using a modified telomeric repeat amplification protocol (TRAP) assay as described previously (47). Sorted cells were lysed in $200 \mu \mathrm{l}$ of ice cold CHAPS lysis buffer and incubated 30 minutes with shaking at $4^{\circ} \mathrm{C}$. The lysate was centrifuged at max speed for 20 minutes at $4^{\circ} \mathrm{C}$, and the supernatant was collected. Telomerase extracts were assessed in 2 steps using a SYBR Green real-time quantitative TRAP assay. First, telomerase-mediated extension of an oligonucleotide trimer (TS) (5'-AATCCGTCGAGCAGAGTT-3') at a final concentration of $900 \mathrm{nM}$, which serves as a substrate for telomerase. Second, hot-start PCR amplification of the resultant product with the oligonucleotide primer pair TS (forward) and CX (reverse) (5'-GTGTAACCCTAACCCTAACCC-3') at a final concentration of $500 \mathrm{nM}$, in $25 \mu 1$ final volume with SYBR Green PCR Master Mix (Life Technologies). Using the Bio-Rad 7300 Thermal cycler, samples were incubated for 20 minutes at $25^{\circ} \mathrm{C}$ for the telomerase reaction followed by a PCR initial activation step at $95^{\circ} \mathrm{C}$ for 10 minutes and amplified in $35 \mathrm{PCR}$ cycles consisting of 30 seconds at $95^{\circ} \mathrm{C}$ (denaturation), 90 seconds at $60^{\circ} \mathrm{C}$ (annealing), and 30 seconds at $72^{\circ} \mathrm{C}$ (extension). Standards and negative controls with heat-inactivated samples and lysis buffer only were assayed on each plate. Each sample was analyzed in at least 3 independent assays. Telomerase activity was calculated relative to that of the 293T cell line and expressed as RTA $\left(\mathrm{Ct}_{293 \mathrm{~T}} / \mathrm{Ct}_{\text {sample }}\right)$.

Statistics. Univariate statistical analysis was performed using GraphPad Prism software. Groups were compared using the nonparametric Kruskal-Wallis or Mann-Whitney tests. Spearman's rank test was used to determine correlations. $P$ values above 0.05 were considered not significant. 
Study approval. The study was approved by the local institutional ethics committee (Comite de Protection des Personnes of the Pitié Salpétrière Hospital, Paris) and all participants provided written informed consent.

\section{Author contributions}

TF designed and performed the research, analyzed data, and wrote the manuscript. VFM designed and performed the research, and analyzed data. TY designed and performed the research. LP, CB, SF, and RZ performed the research. RAK designed the research. JB analyzed clinical data and recruited patients. DS designed and performed the research, analyzed data, and wrote the manuscript. VA designed the research, analyzed data, and wrote the manuscript.

\section{Acknowledgments}

We are very grateful to the patients and staff of the geriatrics department of the Hôpital Pitié Salpêtrière in Paris. We are indebted to Claude Baillou (CIMI INSERM U1135, Paris, France) and Catherine Blanc (Cytometry platform, UPMC, Paris, France) for assistance with cell sorting; and to Sebastien Lemoine (Institut Necker Enfants Malades, Paris, France), Estelle Devevre (Centre d'Imagerie Cellulaire et de Cytométrie from the Cordeliers Research Center, Paris, France), and Anne Laure Bulteau (Institut de Génomique Fonctionnelle, Lyon, France) for analyses with Qlucore Omics explorer software and metabolic immunoassays respectively. We thank Hitoshi Takizawa (IRCMS, Kumamoto, Japan), Mario Pende (Institut Necker Enfants Malades, Paris), and Oliver Bischof (Institut Pasteur, Paris) for constructive discussions, and Justin Frere (University of Arizona, Tucson, Arizona, USA) for English editing. This work was supported by the Fondation pour la Recherche Médicale (FRM) (Project DEQ20120323690), the French Agence Nationale de la Recherche sur le SIDA (ANRS, Project N14007DR), the ANR (Project ANR-14-CE14-0030-01), and the INSERM transversal research program on aging (Project AGEMED).

Address correspondence to: Victor Appay or Delphine Sauce, INSERM Team 'HIV Pathogenesis and Immune Aging', CIMI-Paris, 91 Bd de 1'Hopital, 75013 Paris France. Phone: 33.14.077.8183. Email: victor.appay@ upmc.fr (V. Appay); delphine.sauce@upmc.fr (D. Sauce).

1. Kovtonyuk LV, Fritsch K, Feng X, Manz MG, Takizawa H. Inflamm-aging of hematopoiesis, hematopoietic stem cells, and the bone marrow microenvironment. Front Immunol. 2016;7:502.

2. Geiger H, de Haan G, Florian MC. The ageing haematopoietic stem cell compartment. Nat Rev Immunol. 2013;13(5):376-389.

3. Rossi DJ, et al. Cell intrinsic alterations underlie hematopoietic stem cell aging. Proc Natl Acad Sci USA. 2005;102(26):9194-9199.

4. Chambers SM, Shaw CA, Gatza C, Fisk CJ, Donehower LA, Goodell MA. Aging hematopoietic stem cells decline in function and exhibit epigenetic dysregulation. PLoS Biol. 2007;5(8):e201.

5. Rossi DJ, Bryder D, Seita J, Nussenzweig A, Hoeijmakers J, Weissman IL. Deficiencies in DNA damage repair limit the function of haematopoietic stem cells with age. Nature. 2007;447(7145):725-729.

6. Beerman I, Seita J, Inlay MA, Weissman IL, Rossi DJ. Quiescent hematopoietic stem cells accumulate DNA damage during aging that is repaired upon entry into cell cycle. Cell Stem Cell. 2014;15(1):37-50.

7. Moehrle BM, et al. Stem cell-specific mechanisms ensure genomic fidelity within HSCs and upon aging of HSCs. Cell Rep. 2015;13(11):2412-2424.

8. Flach J, et al. Replication stress is a potent driver of functional decline in ageing haematopoietic stem cells. Nature. 2014;512(7513):198-202.

9. Moehrle BM, Geiger H. Aging of hematopoietic stem cells: DNA damage and mutations? Exp Hematol. 2016;44(10):895-901.

10. Ho TT, et al. Autophagy maintains the metabolism and function of young and old stem cells. Nature. 2017;543(7644):205-210.

11. Kollman C, et al. Donor characteristics as risk factors in recipients after transplantation of bone marrow from unrelated donors: the effect of donor age. Blood. 2001;98(7):2043-2051.

12. Kuranda K, et al. Age-related changes in human hematopoietic stem/progenitor cells. Aging Cell. 2011;10(3):542-546.

13. Pang WW, et al. Human bone marrow hematopoietic stem cells are increased in frequency and myeloid-biased with age. Proc Natl Acad Sci USA. 2011;108(50):20012-20017.

14. Rundberg Nilsson A, Soneji S, Adolfsson S, Bryder D, Pronk CJ. Human and murine hematopoietic stem cell aging is associated with functional impairments and intrinsic megakaryocytic/erythroid bias. PLoS One. 2016;11(7):e0158369.

15. Rübe $\mathrm{CE}$, et al. Accumulation of DNA damage in hematopoietic stem and progenitor cells during human aging. PLoS One. 2011;6(3):e17487.

16. Dykstra B, de Haan G. Hematopoietic stem cell aging and self-renewal. Cell Tissue Res. 2008;331(1):91-101.

17. Cho RH, Sieburg HB, Muller-Sieburg CE. A new mechanism for the aging of hematopoietic stem cells: aging changes the clonal composition of the stem cell compartment but not individual stem cells. Blood. 2008;111(12):5553-5561.

18. Beerman I, et al. Functionally distinct hematopoietic stem cells modulate hematopoietic lineage potential during aging by a mechanism of clonal expansion. Proc Natl Acad Sci USA. 2010;107(12):5465-5470. 
19. Challen GA, Boles NC, Chambers SM, Goodell MA. Distinct hematopoietic stem cell subtypes are differentially regulated by TGF-beta1. Cell Stem Cell. 2010;6(3):265-278.

20. Terstappen LW, Huang S, Safford M, Lansdorp PM, Loken MR. Sequential generations of hematopoietic colonies derived from single nonlineage-committed CD34+CD38 progenitor cells. Blood. 1991;77(6):1218-1227.

21. Galy A, Travis M, Cen D, Chen B. Human T, B, natural killer, and dendritic cells arise from a common bone marrow progenitor cell subset. Immunity. 1995;3(4):459-473.

22. Miettinen M, Lasota J. KIT (CD117): a review on expression in normal and neoplastic tissues, and mutations and their clinicopathologic correlation. Appl Immunohistochem Mol Morphol. 2005;13(3):205-220.

23. Six EM, et al. A human postnatal lymphoid progenitor capable of circulating and seeding the thymus. J Exp Med. 2007;204(13):3085-3093.

24. Florian MC, et al. Cdc42 activity regulates hematopoietic stem cell aging and rejuvenation. Cell Stem Cell. 2012;10(5):520-530.

25. Goodell MA, Brose K, Paradis G, Conner AS, Mulligan RC. Isolation and functional properties of murine hematopoietic stem cells that are replicating in vivo. J Exp Med. 1996;183(4):1797-1806.

26. Morita M, et al. mTOR coordinates protein synthesis, mitochondrial activity and proliferation. Cell Cycle. 2015;14(4):473-480.

27. Miao EA, Rajan JV, Aderem A. Caspase-1-induced pyroptotic cell death. Immunol Rev. 2011;243(1):206-214.

28. Menkova-Garnier I, et al. P2X7 receptor inhibition improves CD34 T-cell differentiation in HIV-infected immunological nonresponders on c-ART. PLoS Pathog. 2016;12(4):e1005571.

29. Sauce $\mathrm{D}$, et al. Lymphopenia-driven homeostatic regulation of naive T cells in elderly and thymectomized young adults. $J$ Immunol. 2012;189(12):5541-5548.

30. Brenchley JM, et al. Expression of CD57 defines replicative senescence and antigen-induced apoptotic death of CD8 ${ }^{+} \mathrm{T}$ cells. Blood. 2003;101(7):2711-2720.

31. Morita M, et al. mTORC1 controls mitochondrial activity and biogenesis through 4E-BP-dependent translational regulation Cell Metab. 2013;18(5):698-711.

32. Rufer $\mathrm{N}$, et al. Telomere fluorescence measurements in granulocytes and T lymphocyte subsets point to a high turnover of hematopoietic stem cells and memory T cells in early childhood. J Exp Med. 1999;190(2):157-167.

33. Mitchell JR, Wood E, Collins K. A telomerase component is defective in the human disease dyskeratosis congenita. Nature. 1999;402(6761):551-555.

34. Vulliamy T, et al. The RNA component of telomerase is mutated in autosomal dominant dyskeratosis congenita. Nature. 2001;413(6854):432-435.

35. Yamaguchi H, et al. Mutations in TERT, the gene for telomerase reverse transcriptase, in aplastic anemia. $N$ Engl J Med. 2005;352(14):1413-1424.

36. Barilari M, et al. ZRF1 is a novel S6 kinase substrate that drives the senescence programme. EMBO J. 2017;36(6):736-750

37. Sauce D, et al. HIV disease progression despite suppression of viral replication is associated with exhaustion of lymphopoiesis. Blood. 2011;117(19):5142-5151.

38. Li G, et al. Decline in miR-181a expression with age impairs T cell receptor sensitivity by increasing DUSP6 activity. Nat Med. 2012;18(10):1518-1524.

39. Briceño O, et al. Reduced naïve CD8(+) T-cell priming efficacy in elderly adults. Aging Cell. 2016;15(1):14-21.

40. Roth A, et al. Telomerase levels control the lifespan of human T lymphocytes. Blood. 2003;102(3):849-857.

41. Najarro K, et al. Telomere length as an indicator of the robustness of B- and T-cell response to influenza in older adults. J Infect Dis. 2015;212(8):1261-1269.

42. Jang YY, Sharkis SJ. A low level of reactive oxygen species selects for primitive hematopoietic stem cells that may reside in the low-oxygenic niche. Blood. 2007;110(8):3056-3063.

43. Chen C, Liu Y, Liu Y, Zheng P. mTOR regulation and therapeutic rejuvenation of aging hematopoietic stem cells. Sci Signal. 2009;2(98):ra75

44. Ocampo A, Reddy P, Belmonte JCI. Anti-aging strategies based on cellular reprogramming. Trends Mol Med. 2016;22(8):725738.

45. Dominguez MH, et al. Highly multiplexed quantitation of gene expression on single cells. J Immunol Methods. 2013;391(12):133-145.

46. Cawthon RM. Telomere length measurement by a novel monochrome multiplex quantitative PCR method. Nucleic Acids Res 2009;37(3):e21.

47. Wege H, Chui MS, Le HT, Tran JM, Zern MA. SYBR Green real-time telomeric repeat amplification protocol for the rapid quantification of telomerase activity. Nucleic Acids Res. 2003;31(2):E3-E3. 